\title{
A Performance Enhancement in A3CovLite Protocol
}

\author{
Abdel-Hamid M.Rashid \\ Department of Information \\ Technology
}

\author{
Hossam Eldeen \\ M.Shamardan \\ Faculty of Computers \& Information
}

\author{
Mostafa-Sami M.Mostafa \\ Helwan University, Egypt
}

\begin{abstract}
The most challenge when designing a topology control protocol is to build a connected reduced topology while extending the sensing coverage area. This paper proposes enhancements to A3CovLite protocol in sensing coverage and energy efficiency issues. Reducing the types of the used messages in the whole network by using two types of messages instead of three as in A3CovLite that lead to enhance the energy saving. Also updating the selection metric to contain the neighbor's average will increase the sensing covered area. Simulation results show a clear enhancement in energy saving that leads to prolong the lifetime of the network and another enhancement in sensing coverage over time.
\end{abstract}

\section{General Terms}

Wireless Sensor Network (WSN), Topology Control (TC), Construction Topology Control.

\section{Keywords}

A3CovLite, Protocol, Connected Dominating Sets (CDS), Energy efficiency, Sensing Coverage, Network Lifetime

\section{INTRODUCTION}

Finding new ways to get data attracts researches and applications today. There is technology to sense, observe, and react to events and phenomena in a specified environment and send it to places where it is analyzed to take the appropriate decision; this technology is based on Wireless Sensor Network (WSN). It's very helpful in many tasks where human interaction can be impossible, risky or even difficult. Wireless sensor networks (WSNs) have been used in a wide range of applications like environment monitoring, military, industrial monitoring and control, etc. They are usually used to support cost-effective sensing in situations where human observation or wired systems deployment can be inefficient, expensive, dangerous, or otherwise untenable.

When deployed the nodes, the initial topology (sometimes called MaxPower Graph) start works. All nodes will transmit at their maximum power. It is not desirable to start the routing process because of: 1) Excess of interference and collision. 2) Reduced capacity. 3) Redundant information: nodes in the same location will sense same events and sending the same data. All these problems will consume the energy rapidly. So it is important to reduce network topology (Minimal Spanning Tree).Topology Control (TC) protocol is one of the most important techniques used in Wireless Sensor Networks for reducing energy consumption and radio interference. A topology control protocol can be defined as an iterative process that dynamically reduces the initial topology of a wireless sensor network through controlling node transmission range with respect to important assets of wireless sensor networks such as connectivity, and coverage $[1,2]$.
Once the initial topology has been established, the TC algorithm performs two iterative phases: the topology construction phase and the topology maintenance (TM) phase. The topology construction phase aims to reduce the exiting initial topology through performing either one of the two operations. The first operation controls nodes' activities such as reducing their transmission power or turning unnecessary nodes off (going to Sleeping status). The second operation controls links activity through disabling or enabling some of them. Once the reduced topology has been constructed, the network starts gathering information from its environment. Every active node participating in the reduced topology carries out many activities that drain its energy so it cannot be active all the time. The TM mechanism should be in place to build a new reduced topology - with the collaboration of formerly inactive nodes - so that all nodes participate in the network, consume their energy in a fair manner, and increase the lifetime of the network.

The topology control (TC) can be described based on the graph theory as follows. Let the graph $G=(V, E)$ denotes the wireless sensor network before running a topology construction algorithm, with $\mathrm{V}$ being the set of sensor nodes, and $\mathrm{E}$ representing the set of communication links. There is a link $(\mathrm{u}, \mathrm{v})$ in $\mathrm{E}$ if and only if the two nodes $\mathrm{u}$ and $\mathrm{v}$ can communicate directly. Running the topology construction algorithm will yield a reduced subgraph $G_{r}=\left(V_{r}, E_{r}\right)$ of $G$ where $V_{r}$ is the set of remaining sensor nodes, and $E_{r}$ is the set of remaining links. The undirected subgraph $G_{r}$ from the original graph $G$ must consider the following constraints: 1) fully distributed: Centralized approaches are failed to perform efficiently in realistic application scenarios. For this reason constructing the topology in a fully distributed fashion is a crucial design challenge. 2) Connectivity: two nodes $u$ and $v$ are connected if there is a path from $\mathrm{u}$ and $\mathrm{v}$, potentially through multiple hops. If two nodes are connected in G, then they should still be connected in $G_{r}$. 3) Localized construction: This reflects the ability of each node $u$ to build its view of the network topology based on the information of all nodes within constant hops of $\mathrm{u}$, usually two or three hops away. Locality implies that the network topology can be easily reconfigured when nodes leave or join the network, and in case of mobility. 4) spanner: for any two nodes $u$ and $v$, if the optimal path between $u$ and $v$ in $G$ has $\cos t c$ then the optimal path between $u$ and $v$ in $G_{r}$ has cost $f(c)$, which is bounded from above by a linear function in c. The Graph $G_{r}$ is called a spanner [3].

The topology construction protocol can be classified into two categories: the first one is connectivity-oriented which provides a connected reduced topology, but does not take into account coverage level of interesting area. This is applied by algorithms like A3 [4], A3Lite [5], EECDS [6], and CDSRule-K [7]. The second category is coverage-oriented which 
guarantees that interesting area will be monitored or sensed by the network as indicated by algorithms like A3Cov [5], ACOS [8], and StanGA[9]. The connectivity-oriented protocols work under assumption: $\mathrm{R}_{\mathrm{C}}=\mathrm{R}_{\mathrm{S}}$; communications radius equal to the sensing radius; but this is not very realistic assumption to make, it dues that many nodes will be put to sleep because they are reachable via communication range but the area where they are may not be covered sufficiently by their sensors.

There are many approaches show how to construct an optimal topology, one approach with controlling transmission power; other approaches use the means of backbones and clusters. The paper focuses on Backbone-Based Techniques which aims to define a chain (backbone) of nodes that guarantee both the connectivity and coverage in the deployment area. This backbone must be formed as relay nodes called Connected Dominating Sets (CDS) [10]. The naive idea to construct a dominated set is creating a spanning tree, which is close to Prim's algorithm [11].

Pedro et al. [5], proposed four simple algorithms that build reduced topologies with very low computational and message complexity without the need of localization information: A3, A3Cov, A3Lite and 3CovLite. If sensing coverage is not as critical as energy, it would be better to use A3Lite, as it needs less number of active nodes and messages. If sensing coverage is very important for the application, then the A3CovLite is the best option mostly because of the lower message complexity.

The paper introduces an enhancement for A3CovLite protocol, which is coverage-oriented protocol. The performance of the enhanced protocol is compared to the original A3CovLite. The results demonstrate that the enhanced protocol increases the sensing coverage area and it's more efficient in energy saving.

This paper is organized as follow: in section 2; overview of original A3Covlite protocol, in section 3; proposed enhanced protocol is described in details, in section 4; experiments and simulation results of the proposed protocol compared to its original A3CovLite protocol and in Section 5; conclusion.

\section{OVERVIEW OF A3COVLITE PROTOCOL}

\subsection{Description of A3CovLite Protocol}

A3CovLite protocol [5] is coverage-oriented protocol which provides better sensing coverage of the area. The A3CovLite protocol assumes no prior knowledge in regards to the position or orientation of the nodes. The algorithm uses messages to determine the neighbor's distance and their energy level based on the strength of those sent and received messages. These calculated energy and distance characteristics are saved by each node as its selection metric. The used messages are categorized into three types: Hello message, Parent Recognition message, and Sensing Coverage message. The message complexity of this protocol is at most $3 \mathrm{n}$ in the case that all nodes are selected to be active. The following steps detailed the A3CovLite protocol:

Step a) preselected node, let say node $x$, starts the tree creation process by sending a Hello message. Each Hello message includes the ID of the parent node, except in the case of the sink which in that field will have its own ID.

Step b) when the neighbors of node $x$ receive the message, they do the following tasks:
- Change their status to Waiting Active.

- Calculate the selection metric of (Equation 1).

- Register the sender as its parent node.

- Each node sets timeout A inversely proportional to their selection to send a Hello message.

- Set timeout B, half value of timeout A, to send a Parent Recognition message.

- Evaluates if it is sensing-covered by its parent node.

Step c) during the Waiting Active state several events may happen:

- The node may receive a Parent Recognition message from one of its brothers (all nodes under the area of coverage of the same parent node). In this case, the receiver node cancels timeout $B$ and remains in the Waiting Active state.

- The node may receive a Hello message from a nonbrother, in which case the node resets timeout A to its original value and remains in the Waiting Active state. This means that a non-brother node started a neighborhood discovery process and needs some time to explore and let its branch grow to cover as many nodes as possible, decreasing the probability of the receiver node having unvisited nodes in its neighborhood and becoming active.

- Timeout A may expire. When this happens, the node sends a Hello message and goes to the Active Candidate state.

- Timeout B may expire. In this case, the node sends a Parent Recognition message and remains in the Waiting Active state.

- The node may receive a Hello message from a brother, in which case the node goes to the Sleep Candidate state. Upon reaching this state, the node turns the radio off temporarily, cancels timeout $\mathrm{A}$ and sets timeout $\mathrm{C}$. When timeout $\mathrm{C}$ expires, the node will wake up and start its Second Opportunity Process, in which the node goes to the Active Candidate state to explore its neighborhood for unvisited nodes.

Step d) once the node is in the Active Candidate state, it sends a Hello message and sets timeout D in order to wait for Parent Recognition message from its children.

- If the Active Candidate receives at least one Parent Recognition message from a child, then the node goes to the Active Node state, which means, that it will be part of the CDS tree and send a Sensing Coverage message to its neighbors in order to notify that it has been selected to be active.

- If the Active Candidate does not receive any messages of kind Parent Recognition, it means that it does not provide connectivity to any node, so it is verifying if the node was sensing-covered by any other node. In the case where the node was sensing-covered, the node will be sent to the Sleeping Node mode; otherwise, the node will change its state to Active Node and send a Sensing Coverage message to its neighbors in order to notify that it has been selected to be active. 


\subsection{The Mathematical Statement for the Selection Metric}

Equation 1 [6] calculates the selection metrics as proportional to the remaining energy of the node and the distance from the parent node. This equation gives priority to those nodes of a distance far away from the parent node with a higher remaining energy, in order to build a tree with fewer nodes and better coverage.

$$
\mathbf{M}_{x, y}=W_{E} \cdot\left(\frac{E_{x}}{E_{\max }}\right)+W_{D} \cdot\left(\frac{R_{R S I}}{R_{S S I^{*}}}\right)
$$

Where $x$ is a candidate node, $\mathrm{y}$ is a node inviting $x, W_{E}$ is the weight for the remaining energy in the node, $W_{D}$ is the weight for the distance from the parent node, $E_{x}$ is the remaining energy in node $x, E_{\max }$ is the threshold that defines the maximum energy of a node, $R S S I_{y}$ is the Received Signal Strength Indicator from the parent node, and $R S S I^{*}$ is the minimum RSSI to ensure connectivity, which is given by the sensitivity of the receiver. Equation 1 produces a value between 0 and 1 . The higher the metric's value means the higher the chance for that node to be a candidate for joining the growing tree.

\section{PROPOSED ENHANCED PROTOCOL}

\subsection{Description of the Proposed Enhanced Protocol}

The following two main operations explain simply what happen in the proposed enhanced protocol:

A- Sending Hello Message

$B$ - Receiving Hello Message

\section{A- Sending Hello Message:}

When a node start sending Hello message to its neighbors in its range transmission; this node may be initiator node (sink node) that start the tree creation process, or node in active candidate state; the sender node broadcasts Hello message that includes ID of the parent to all neighbors then it waits timeout A for listening Hello message from one or more of its children.

\section{B- Receiving Hello Message:}

When a node or more receive Hello message from the Sender, there are many cases to it according its state:

- If the receiver nodes in the initial state it will do the following tasks:

- Change their status to Waiting Active.

- Calculate the selection metric of (Equation 2).

- Register the sender as its parent node.

- Each node sets timeout B inversely proportional to their metric selection to send a Hello message.

- Evaluate if it is sensing-covered by its parent node.

- If the receiver node in Waiting Active state; there are two cases:

- It may receive a Hello message from a non-brother, in which case the node resets timeout $B$ to its original value and remains in the Waiting Active state. This means that a non-brother node started a neighborhood discovery process and needs some time to explore and let its branch grow to cover as many nodes as possible, decreasing the probability of the receiver node having unvisited nodes in its neighborhood and becoming active.

- It may receive a Hello message from a brother, in which case the node goes to the Sleep Candidate state. Upon reaching this state, the node turns the radio off temporarily, cancels timeout $\mathrm{B}$ and sets timeout $\mathrm{C}$. When timeout $\mathrm{C}$ expires, the node will wake up and start its Second Opportunity Process, in which the node goes to the Active Candidate state to explore its neighborhood for unvisited nodes.

- If the receiver node in the Active Candidate state, and receives a Hello messages from one or more of its children (the ID of receiver as the parent ID of the sender), then the node goes to the Active Node state, which means, that it will be part of the CDS tree and send a Sensing Coverage message to its neighbors in order to notify that it has been selected to be active.

- If the Active Candidate node does not receive any Hello message from any of it children during timeout $\mathrm{A}$, it means that it does not provide connectivity to any node, so it is verifying if the node was sensing-covered by any other node. In the case where the node was sensing-covered, the node will be sent to the Sleeping Node mode; otherwise, the node will change its state to Active Node and send a Sensing Coverage message to its neighbors in order to notify that it has been selected to be active.

\subsection{The Proposed Enhanced Selection Metric}

The final metric is calculated using equation 2 :

$$
M_{x, y}=W 1 \cdot\left(\frac{E_{x}}{E_{\max }}\right)+W 2 \cdot\left(\frac{R S S I_{y}}{R S S I^{*}}\right)+W 3 \cdot \operatorname{avg}(N e)_{x}
$$

Where $x$ is a candidate node, $\mathrm{y}$ is its parent, $W 1$ is the weight for the remaining energy in the node, $W 2$ is the weight for the distance from the parent node, $W 3$ is the weight for the neighbors' average for node $x, E_{x}$ is the remaining energy in node $x, E_{\max }$ is the maximum initial energy, $R S S I_{y}$ is the Received Signal Strength Indicator from the parent node or in other words the distance between the node $x$ and $y$, and $R S S I^{*}$ is the minimum RSSI accepted to ensure connectivity with a good signal level, preferably several $\mathrm{dBms}$ above the noise level, $\operatorname{avg}(\mathrm{Ne})_{x}$ is calculated according the following equation 3:

$\operatorname{avg}(N e)_{x}=\frac{(\text { total neighbors of node } X)}{(\text { total nodes in the network })}$

Equation 2 produces a value between 0 and 1 . The higher the metric's value means the higher the chance for that node to be a candidate for joining the growing tree.

In equation 2 ; it must be considered that:

- $\quad W 1+W 2+W 3=1$

- Depending on remaining energy as the only selection metric will support the reliability of the network but produce more active nodes that consume more energy, also non-covered area occurred due to continuously selecting of the highest remain energy nodes in next level to sink which will die after few rounds, causing fall the network lifetime down although there are some nodes has 
energy in the far levels.

- Depending on the distance as the only selection metric will reduce the number of hops to enhance the network coverage but produce low energy nodes may be included in the tree, which reducing reliability because the early failures of nodes, and increasing the TM invocations number, and also reducing the lifetime of the tree.

- So, the most appropriate choice is to weight energy metric and distance metric equally. To maintain the reliability of the tree and reduce TM invocations to stop wasting the energy.

- In this proposed Enhancement, adding third metric; neighbors' average; it has two benefits:

1) It acts as resolving factor in case the equality of selection metric between more than one nodes.

2) It helps to choose the best active nodes that have more number of neighbors around it so it can serve them in sensing issues.

- After experiments with different values of W1, W2 and W3, it is found the following orders of the three metrics (energy, distance and neighbors' average) are more suitable to test the effect of neighbors' average metric without affecting the reliability of the tree in addition to get better results; the orders will be: 1) order1 $(0.4,0.3$, and 0.3$)$. 2) order $2(0.3,0.4$, and 0.3$)$. The two orders will be used in the following experiments in this paper.

\subsection{Similarities and Differences to Original A3Covlite}

- As original A3CovLite and A3family protocols, the proposed one guarantees the connectivity property described in Lemma 1 [5].

- Lemma 1: If the initial graph is connected, the reduced graph is also connected.

So every node is forced to send a Hello message, each node explores all its neighbors from the original graph looking for unvisited nodes. The algorithm continues until all nodes are visited in the total area of coverage of the tree exploring all edges from this set of nodes. This means that the final tree is a connected subgraph itself because it has no edge to unvisited nodes. If exists an uncovered node it means that there is no edge between any of the covered nodes and the uncovered node. Then the initial graph cannot be connected because it has at least two non-connected sets of nodes, which contradicts the initial assumption of a connected graph.

- As original A3CovLite idea, a node is sensing-covered if $\forall \mathrm{x}, \mathrm{y} \in \mathrm{V}, \mathrm{d}(\mathrm{x}, \mathrm{y}) \leq \alpha \mathrm{R}_{\text {sense }} \quad$ where $0<\alpha<$ $\frac{\mathrm{R}_{\text {comm }}}{\mathrm{R}_{\text {sense }}}$

The node is considered sensing-covered, and goes to the Sleeping Node state immediately. if $d(x, y) \leq \alpha R_{\text {sense }}$ it means that the receiver node is under the communication coverage of the sender but not within its sensing coverage, so the message is ignored and the receiver node keeps waiting for messages until the timeout expires.

- Different from original A3CovLite selection metric, it considers the neighbors' average with distance and remains energy.

- As original A3CovLite, the selection metric is simple, requires only one hop information.
- Different from original A3CovLite in messages overhead, the proposed used two types: Hello message, and Sensing Coverage message. The message complexity of this protocol is at most $2 \mathrm{n}$ in the case that all nodes are selected to be active. But the original use three types of messages: Hello message, Parent Recognition message and Sensing Coverage message with complexity $3 n$ in the case all nodes are selected. So it is less complexity than the Original A3CovLite.

\section{The EXPERIMENTS AND SIMULATION RESULTS}

\subsection{The Performance Evaluation Method}

In this section; the performance of the enhanced protocol was compared to its original A3Covlite protocol. The experiments were applied to evaluate the new optimizations in A3Covlite protocol. Firstly, evaluating the impact of adding neighbor's average metric. Secondly, evaluating the impact of using two types of messages instead of three types. Finally, to evaluate the impact of using these two types of messages in conjunction with neighbor's average metric.

The experiments run on an event-driven simulation tool named Atarraya which was developed for teaching, researching and evaluating topology control protocols. When using the Atarraya and performing the experiments, the following assumptions made: 1) Nodes are located in a two dimensional space and have a perfect communication and sensing coverage disk. 2) Nodes have no information about their position, orientation, or neighbors.3) the initial graph, the one formed right after the deployment, is assumed to be connected.4) Distances can be calculated as a metric perfectly proportional to the Received Signal Strength Indicator (RSSI). 5) There is no packet loss at the Data Link Layer.

Also the energy model used in the simulation is MICA2 $\mathrm{X}$-Bow Mote, its energy consumption specifications in table1. DGTTRec algorithm [2] was selected as topology maintenance technique; it is invoked every time a threshold (e.g., a time or energy threshold) is exceeded, it terminates the current reduced topology and calls the topology construction algorithm to create a new one. Table 1 predefined the experiments' parameters.

Table 1: Simulation Parameters

\begin{tabular}{|c|c|c|c|}
\hline Parameter & Exp 1 & Exp 2 & $\operatorname{Exp} 3$ \\
\hline Number of Nodes & $\begin{array}{c}80,100,200 \\
\text { (immobile nodes) }\end{array}$ & $\begin{array}{c}300,400 \\
\text { (immobile nodes) }\end{array}$ & $\begin{array}{c}600,800 \\
\text { (immobile nodes) }\end{array}$ \\
\hline Deployment Area & $200 \times 200 \mathrm{~m}$ & $400 \times 400 \mathrm{~m}$ & $600 \times 600 \mathrm{~m}$ \\
\hline $\begin{array}{c}\text { Node Location } \\
\text { Distribution }\end{array}$ & Uniform $(200,200)$ & Uniform $(400,400)$ & Uniform $(600,600)$ \\
\hline Number of Sinks & \multicolumn{3}{|c|}{1 sink node (in the center) } \\
\hline \begin{tabular}{|c|}
$\begin{array}{c}\text { Max Transmission } \\
\text { Range }\end{array}$ \\
\end{tabular} & \multicolumn{3}{|c|}{$40 \mathrm{~m}$} \\
\hline Sensing Range & \multicolumn{3}{|c|}{$20 \mathrm{~m}$} \\
\hline $\begin{array}{c}\text { Critical Time } \\
\text { Threshold } \\
\end{array}$ & \multicolumn{3}{|c|}{7200 seconds ( 2 hours) } \\
\hline Initial Energy & \multicolumn{3}{|c|}{$3200 \mathrm{~mA}-\mathrm{h}$ (milliampere-hour) } \\
\hline $\begin{array}{l}\text { Node Energy } \\
\text { Distribution }\end{array}$ & \multicolumn{3}{|c|}{ Uniform } \\
\hline \begin{tabular}{|c|}
$\begin{array}{c}\text { Number of random } \\
\text { scenarios }\end{array}$ \\
\end{tabular} & \multicolumn{3}{|c|}{20} \\
\hline \multirow{5}{*}{ Energy model } & \multicolumn{3}{|c|}{ MICA2 X-Bow Mote } \\
\hline & Processor & Active $=8 \mathrm{~mA}$ & Sleep $=15 \mu \mathrm{A}$ \\
\hline & Sensor & Active $=5 \mathrm{~mA}$ & Sleep $=5 \mu \mathrm{A}$ \\
\hline & \multirow{2}{*}{ Radio } & \multicolumn{2}{|c|}{ Tx_Consumption $=12 \mathrm{~mA}$} \\
\hline & & Rx_Consumption $=7 \mathrm{~m}$ & A $\quad$ Sleep $=1 \mu \mathrm{A}$ \\
\hline Message length & \multicolumn{3}{|c|}{ Short: 40 bytes, Long: 100 bytes } \\
\hline
\end{tabular}


The comparison performance between the enhanced protocol and its original A3CovLite protocol is evaluated in terms of: 1) the number of active nodes which guarantees the whole network's coverage; it has a direct impact on the lifetime of the network. 2) Number of sent messages, which are related with the scalability of the protocol and the energy consumption. 3) The ratio spent energy in the topology construction process.4) sensing coverage area. 5) The network's lifetime: it is the period of time which the network stays alive.

\subsection{Experiment 1: Impact of neighbor's average metric in Active nodes number}

The scenarios were performed at the enhanced protocol in case order1, order2, and at the original A3CovLite protocol when parameters: network density $(80,100,300,600$, and $800)$, $\alpha$ coverage value equal to 1.3 .

Figure 1 shows there is equality or low increasing compared to original A3CovLite. When weight is order1, there is equality in active nodes in case the network density 80 . While one active node increases in case the network density 100 and 300; then there is a little increase (maximum four active nodes) in case the network density 600; and two active nodes increase in case the network density 800 .

Also Figure 1 shows there is low increasing in the number of active nodes when weight is order2. In case the network density 80 there is one active node increases. While three active nodes increase in case the network density 100; then there are seven active nodes increase in case the network density 300; and five active nodes increase in case the network density 600 and 800 .

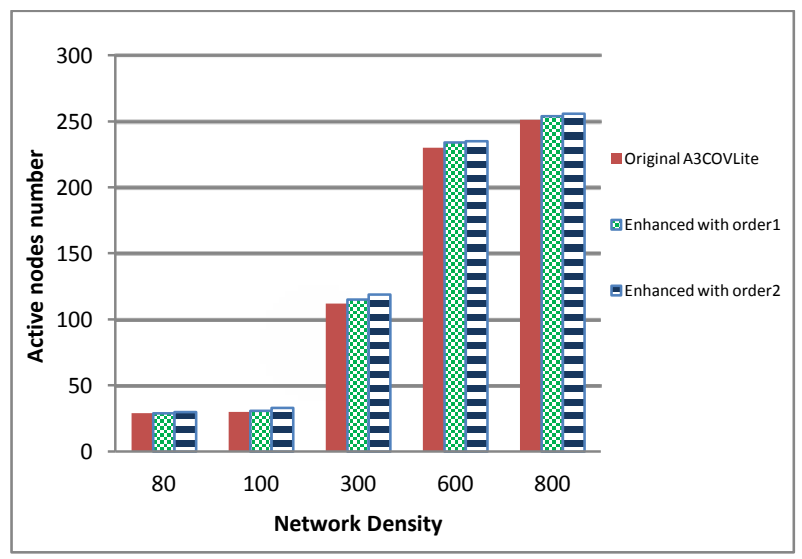

Figure 1: Number of Active Nodes

From the results, the operator can use the two metrics (distance metric and neighbor's average metric) as one bundle metric beside the main metric of the remain energy in the node. He can use same heaviness weights to distance and neighbor's average to have nearly equality in active nodes number as original A3CovLite as it was produced from enhanced order1.The benefit from chosen active nodes based on the bundle metric is the selected active nodes will be more quality due to:

- They will serve more nodes in its neighborhood area considering feature of far distance from parent node which leads improving in sensing coverage as it will be discussed in section 4.4 .

- Keeping the energy metric's weight larger than the other weights of bundle metric is the best choice; it protects the nodes in the next level to the sink from early death which causes a hole (uncovered area) between the sink and the nodes in the next levels. that's fallen the network lifetime down although there are some nodes have an energy in these far levels.

\subsection{Experiment 2: Impact of using two types of messages}

The scenarios were performed at the enhanced protocol (without neighbor's average metric) and at the original A3CovLite protocol when following parameters are set: network density $(100,200,400$, and 800), $\alpha$ coverage value equal to 0.7. Note; the enhanced protocol produces the same number of active nodes as the original A3CovLite protocol.

Figure 2 shows an enhancement in the proposed protocol. The enhanced protocol outperforms A3CovLite in reducing the number of sent messages.

The previous evaluation shows that using two types of messages: Hello message and Sensing Covered message in the enhanced protocol instead of three types of messages as the original A3CovLite protocol that will decrease the total number of sent messages and relatively decrease the total spend energy consumed that cause the lifetime of the network to be prolonged.

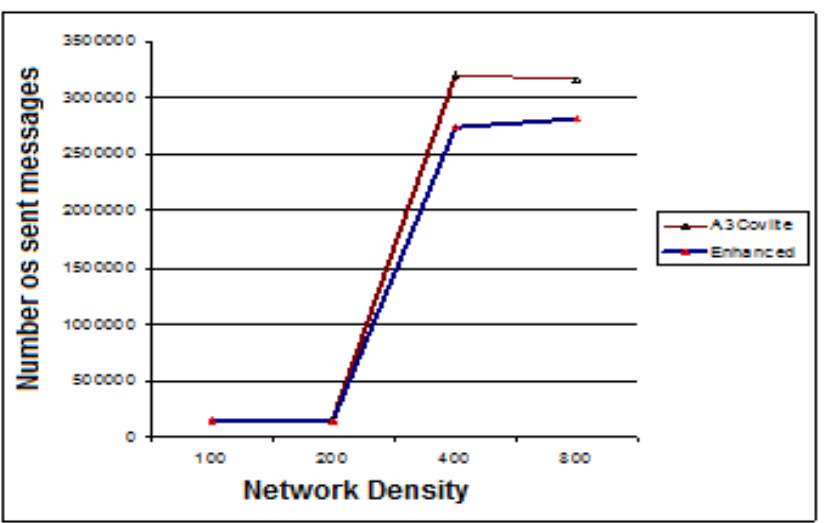

Figure 2: Number of Sent messages

Also Figure 3 shows that the enhanced protocol outperforms original A3CovLite protocol in energy efficiency at all network densities. It is relevant to the decrease of the sent messages number.

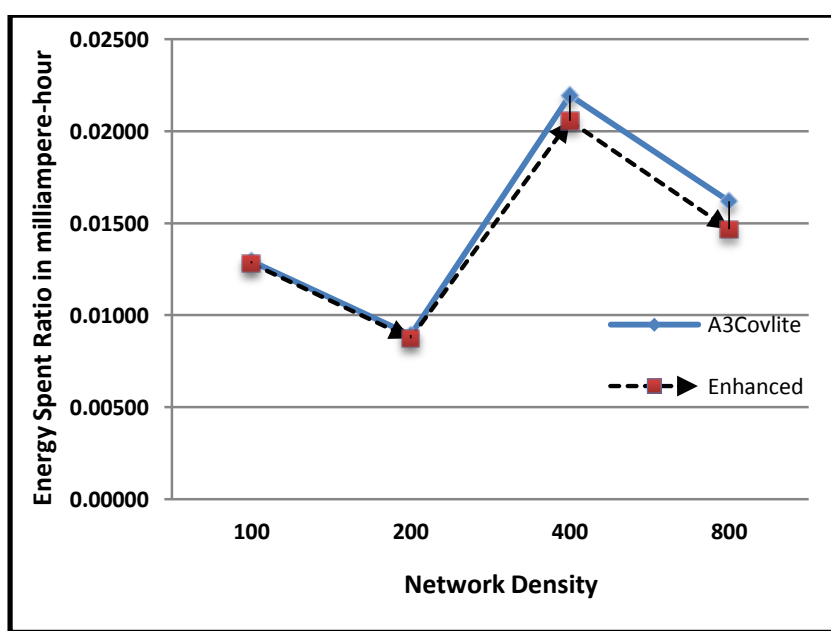

Figure 3: Energy Spent Ratio 


\subsection{Experiment 3: Impact of using two types of messages and neighbor's average metric together}

The scenarios were performed at the enhanced protocol and at the original A3CovLite protocol in conjunction with DGTTRec, when parameters: network density $(80,100,300$, 600 and 800$)$, $\alpha$ coverage value equal to 0.7 . Note; the applied enhanced protocol has two features: 1) it has two types of messages as it is explained in section 4.3. 2) it uses order1 as it is explained in section 4.2.

Figure 4 and figure 5 show there is a clear improvement in sensing coverage ratio over time compared to A3CovLite protocol. This outperform is related to the produced active nodes from the enhanced protocol with order1; these active nodes have more quality in sensing coverage issues.

Also figure 6 show there is an extent in the network's lifetime compared to original A3CovLite. This prolongation in the network's lifetime results from using less sent messages number that relatively decrease energy consumption rate.

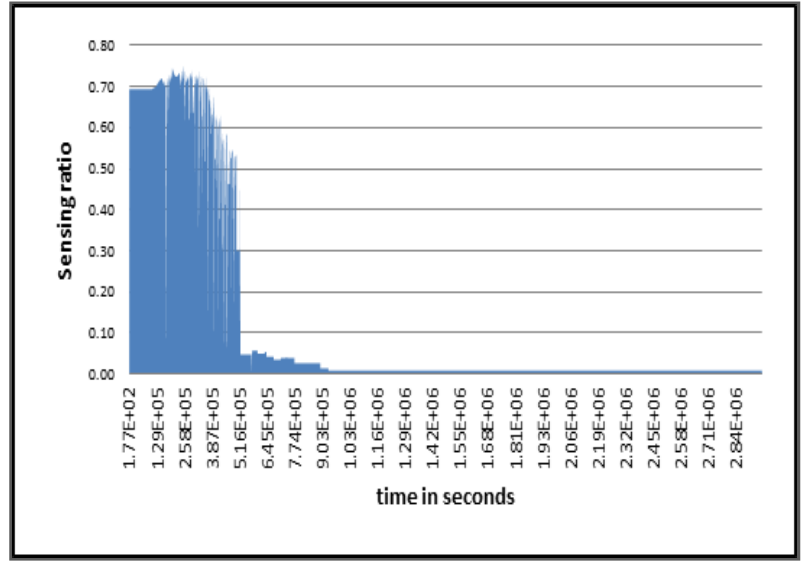

Figure 4: Sensing ratio for A3CovLite when 300 nodes density

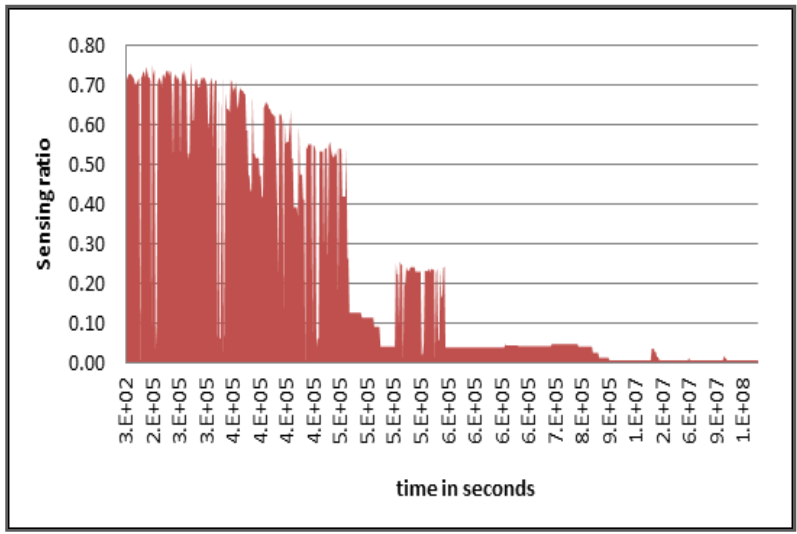

Figure 5: Sensing ratio for enhanced protocol when 300 nodes density

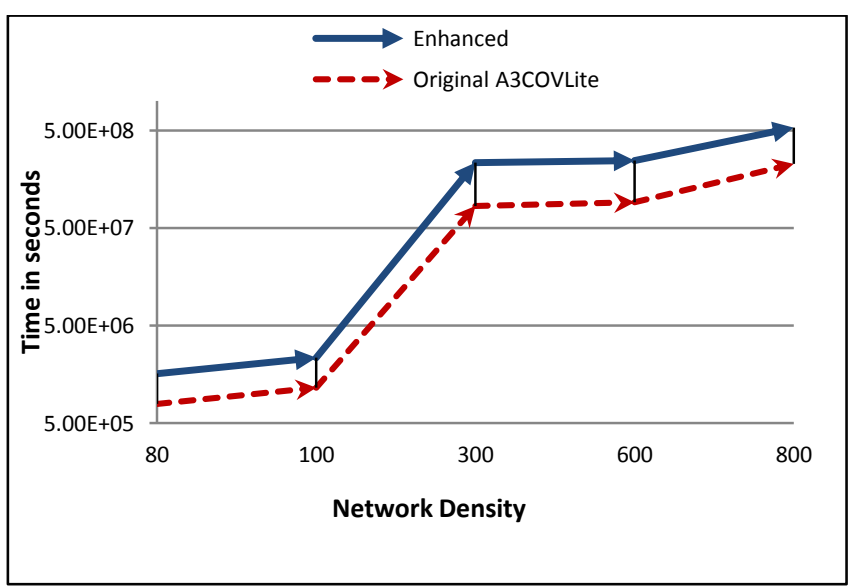

Figure 6: The lifetime of the WSN

\section{CONCLUSION}

In this paper, enhancements to the A3CovLite protocol are introduced, these proposed enhancements are:

- Adding a third metric "neighbor's average" beside the energy and distance metrics to improve the quality of selected active nodes which will be members in the CDS tree which will improve the coverage sensing issues.

- Introducing two orders for the weights in the selection metric. The two orders do not affect the network reliability and extend the lifetime of the network.

- Using two types of messages instead of three messages to improve the energy efficiency.

From the experimental results, the enhanced protocol is better in energy efficiency and it extends the network's lifetime compared to original A3CovLite protocol; in addition it increases the sensing covered area overtime. In future it is recommended to reduce the packet size of the Hello message to provide more energy efficiency. Also it is recommended to consider heterogeneous networks while evaluating the performance of the enhanced protocol to get more realistic results.

\section{REFERENCES}

[1] Paolo.Santi, "Topology control in wireless ad hoc and sensor networks", ACM Computing Surveys, Vol. 37, no. 2, 2005, pp. 164-194.

[2] Pedro M. Wightman, Miguel A. Labrador , "Topology Control in Wireless Sensor Networks - with a companion simulation tool for teaching and research", Springer, 2009.

[3] Nedal Ababneh," Performance Evaluation of a Topology Control Algorithm for Wireless Sensor Networks", International Journal of Distributed Sensor Networks, Volume 2010 (2010), Article ID 671385.

[4] Pedro M. Wightman, Miguel A. Labrador,"A3: a topology control algorithm for wireless sensor networks", In Proceedings of the IEEE GLOBECOM, New Orleans, USA, November 2008.

[5] Pedro M. Wightman, Miguel A. Labrador,"A family of simple distributed minimum connected dominating setbased topology construction algorithms", Journal of 
Network and Computer Applications 34 (2011) 19972010.

[6] Zeng Yuanyuan, , Xiaohua Jia, He Yanxiang, "Energy efficient distributed connected dominating sets construction in wireless sensor networks", in Proceeding of the ACM International Conference on Communications and Mobile Computing, 2006, pp. 797802.

[7] Jie Wu, , Fei Dai, “An extended localized algorithm for connected dominating set formation in ad hoc wireless networks", IEEE Trans. Parallel Distributed Syst. 15(10), 2004, pp. 908-920.

[8] Yanli Cai, Minglu Li, Wei Shu, and Min-You Wu, "Acos: an area-based collaborative sleeping protocol for wireless sensor networks", Ad Hoc \& Sensor Wireless
Networks 2007; (1): 77-97.

[9] Hongxing Bai, Xi Chen, Bin Li, Dianfei Han. "A location-free algorithm of energy-efficient connected coverage for high density wireless sensor networks", Discrete Event Dynamic Systems 2007;17(1):1-21.

[10] S. Guha and S. Khuller, "Approximation algorithms for connected dominating sets", in Proceedings of 4th Annual. European Symposium on Algorithms (ESA), Springer, Vol. 1136 of Lecture Notes in Computer Science, 1996, pp. 179-193.

[11] R. C. Prim, "Shortest connection networks and some generalizations", Bell Syst. Tech. J., 1957, pp.13891401. 